\title{
Endosonography for mediastinal disease: esophageal ultrasound vs. endobronchial ultrasound
}

Authors

Institution
Nikhil Meena, Thaddeus Bartter

University of Arkansas for Medical Sciences and Central Arkansas Veterans Healthcare System, Little Rock, Arkansas, United States submitted

12. December 2014 accepted after revision 23. March 2015

\section{Bibliography}

DOI http://dx.doi.org/

10.1055/s-0034-1392092

Published online: 21.5.2015

Endosc Int Open 2015; 03:

E302-E306

(c) Georg Thieme Verlag KG

Stuttgart · New York

E-ISSN 2196-9736

\section{Corresponding author}

\section{Dr. Nikhil Meena}

University of Arkansas for

Medical Sciences

4301 W. Markham Mail Slot \#555

Little Rock, Arkansas 72205

United States

Fax: +1-501-686-7893

nkmeena@uams.edu
Background and study aims: One can approach mediastinal pathology via esophageal ultrasound (EUS) and/or endobronchial ultrasound (EBUS). It has been suggested that EUS is better tolerated by patients. If so, EUS might be the procedure of choice when suspect lesions are accessible via EUS.We studied procedural characteristics of EUS with fine needle aspiration (EUS-FNA) and EBUS with transbronchial needle aspiration (EBUS-TBNA) to see how they differed.

Patients and methods: Retrospective review of consecutive EBUS and EUS procedures performed on patients over nine months. One hundred fiftyfive procedures were analyzed (61 EUS, 73 EBUS, 21 EUS+EBUS). For EUS, EBUS, and EUS+EBUS, $1.4,2.0$ and 2.5 sites (mean) were sampled, respectively. EUS required approximately onehalf of the time of EBUS or the combined procedures; 13.1 vs. 24.1 and $26.9 \mathrm{~min}$, respectively

\begin{tabular}{ll}
\hline Abbreviations \\
$\nabla$ \\
EBUS & endobronchial ultrasound \\
EUS & esophageal ultrasound \\
FNA & fine needle aspiration \\
ROSE & rapid on-site cytologic evaluation
\end{tabular}

\section{Introduction}

$\nabla$

In 2005, Dr. Vilmann, a gastroenterologist and leader in the field of esophageal ultrasound (EUS), who did some of the pioneering work on esophageal access of mediastinal structures for the diagnosis and staging of thoracic diseases, stated: "But how can we proceed to implement EUSFNA as a routine procedure in respiratory medicine? Now most groups performing EUS-FNA in the chest are still gastroenterologists, because the method was originally developed in this specialty" [1]. This quote is as relevant today as it was then; it has subsequently been shown that pul-
$(P<0.0001$ for EUS vs. both EBUS and EUS+EBUS). Sedation dosing was statistically lower for EUS and not significantly different between EBUS and the combined approach. EUS also involved lower oxygen requirements and shorter time to discharge. Because fewer mean sites were sampled with EUS than with EBUS or the combined procedure, we performed analysis restricted to procedures that involved sampling of $\leq 2$ sites to determine whether approach-related differences in procedure characteristics were preserved. There were 56 such EUS procedures and 52 such EBUS procedures. EUS remained significantly faster and required less patient sedation.

Conclusions: EUS involved statistically significant economies of time and sedation. This has implications with respect to safety and productivity. When applicable, EUS is the procedure of choice.

monologists can effectively use the esophagus alone or simultaneously with airway access using the endoscope originally designed for bronchoscopic ultrasound and that pulmonologists can be trained to use the endoscope used by gastroenterology [2-7], but EUS for the diagnosis of mediastinal disease is not widely performed by pulmonologists and is, in fact, discouraged by some of them [8]. There have been few studies of performance characteristics of EUS vs. EBUS. In our practice, we perform both EBUS and EUS and the approach chosen is based upon patient anatomy. We performed a retrospective review of our database specifically to contrast EBUS with EUS and to investigate relationships between route of access and other procedural parameters. 
Table 1 Data for all treatment groups: EUS, EBUS, and EUS + EBUS.

\begin{tabular}{|c|c|c|c|c|c|c|}
\hline & $\begin{array}{l}\text { EUS } \\
(n=61)\end{array}$ & $\begin{array}{l}\text { EBUS } \\
(n=73)\end{array}$ & $\begin{array}{l}\text { EBUS+EUS } \\
(n=21)\end{array}$ & $\begin{array}{l}P \text { value } \\
\text { EUS vs. EBUS }\end{array}$ & $\begin{array}{l}P \text { value } \\
\text { EUS vs. EBUS + EUS }\end{array}$ & $\begin{array}{l}P \text { value } \\
\text { EBUS vs. EBUS + EUS }\end{array}$ \\
\hline Age (years) & $59.4 \pm 14.5$ & $61.7 \pm 13.6$ & $61.7 \pm 13.7$ & 0.36 & 0.78 & 0.71 \\
\hline Total sites & $1.4 \pm 0.6$ & $2.0 \pm 0.8$ & $2.5 \pm 0.9$ & $<0.0001$ & $<0.0001$ & 0.03 \\
\hline Midazolam (mg) & $3.4 \pm 1.4$ & $4.6 \pm 1.9$ & $4.3 \pm 1.8$ & 0.0002 & 0.001 & 0.59 \\
\hline Fentanyl $(\mu \mathrm{g})$ & $73.6 \pm 27$ & $98.3 \pm 39.7$ & $97.6 \pm 35.3$ & 0.0001 & 0.002 & 0.94 \\
\hline Procedure time (min) & $13.1 \pm 5.1$ & $24.1 \pm 8.4$ & $26.9 \pm 8.4$ & $<0.0001$ & $<0.0001$ & 0.19 \\
\hline $\mathrm{O}_{2}$ flow (L/min) & $5.3 \pm 2.6$ & $6.7 \pm 3.4$ & $5.8 \pm 2.8$ & 0.009 & 0.43 & 0.27 \\
\hline Time to room air (min) & $18.9 \pm 19.7$ & $26.5 \pm 23.9$ & $35.5 \pm 28.8$ & 0.05 & 0.005 & 0.15 \\
\hline Time to discharge ( $\mathrm{min}$ ) & $42.7 \pm 15.9$ & $55.7 \pm 20.6$ & $60.8 \pm 21.9$ & 0.0002 & 0.0002 & 0.33 \\
\hline
\end{tabular}

Abbreviations: EUS, esophageal ultrasound; EBUS, endobronchial ultrasound.

\section{Methods}

$\nabla$

After institutional review board approval, all ultrasound-guided endoscopies performed by Interventional Pulmonary at a single institution from August 1, 2012 until April 30, 2013 were retrospectively reviewed. To allow head-to-head comparison of EUS and EBUS procedures, patients requiring sedation for additional procedures such as regular bronchoscopy or chest tube insertion were excluded. All procedures were performed by one or both of the authors, both of whom are pulmonologists. At our institution, the endoscope that was originally designed for EBUS (BF-UC180F, Olympus Medical Supply Corporation, Melville, New York, United States) was used for both EBUS and EUS; however, if indicated, crossover from one route of access to the other was readily possible. (We limited our use of both techniques to the diagnosis of thoracic disease; endosonography for primary gastrointestinal processes was performed by gastroenterology.) Positioning for both (EBUS and EUS) was the same. Patients were supine on a stretcher with the head elevated to about 30 degrees. All sampling was performed with the 21-gauge needle made by Olympus for the BF-UC180F endoscope. Suction was routinely applied. The number of punctures was determined by on-site adequacy evaluation and by the level of suspiction for malignant involvment, and if so, the suspect lesions were sampled up to ten times. However, a standard of at least three passes was performed on all lesions. Rapid on-site cytologic evaluation (ROSE) was available for all procedures.

The following parameters were selected for comparison: type of procedure (EUS, EBUS, EUS+EBUS), sedation dosing, number of sites biopsied, specific sites biopsied, yield, procedure time, maximal oxygen flow during the procedure, time on oxygen postprocedure, and total time from procedure termination (scope out) until discharge. Student's $t$-tests were used to determine significance with $P<0.05$ considered significant.

\section{Results}

\section{$\nabla$}

One hundred and sixty-five ultrasound-guided needle aspiration biopsy procedures were performed over the study interval. For three of these procedures, propofol was used for sedation, making it impossible to do comparative analysis. For seven procedures, the relevant data were not available on retrospective chart review. These ten procedures were eliminated from the analysis, leaving 155 patient procedures for review. There were 61 patients who underwent EUS alone, 73 patients who underwent EBUS alone, and 21 patients who underwent combined procedures. Age distribution, drug doses administered, procedure times, oxygen flow rates, and recovery times are presented in - Table 1. One hundred and fifty of the procedures were performed for both diagnosis and staging of lung cancer. The remaining five procedures were performed to evaluate for possible mediastinal metastases of cancers previously diagnosed. These procedures were performed on four patients diagnosed with adenocarcinoma of the lung previously diagnosed using CT-guided biopsy and one patient diagnosed with adenocarcinoma of the breast. The midazolam dosing for EUS alone was lower than for EBUS alone $(P<0.0002)$ and for the combined procedure $(P<$ 0.001 ). There was no significant midazolam dosing difference between the EBUS and EUS+EBUS groups. The same pattern was found for fentanyl dosing; fentanyl dosing for EUS alone was lower than for EBUS alone $(P<0.0001)$ and for the combined procedure $(P<0.002)$, although there was no significant fentanyl dosing difference between the EBUS and EUS+EBUS groups. Mean number of sites sampled varied significantly between groups and for EUS vs. the other two groups $(P<0.0001)$. The mean procedure time for EUS was approximately one-half the mean procedure time required for EBUS $(P<0.0001)$. Procedure times for EBUS and EBU+EUS were not significantly different. Mean maximal oxygen flow rate was lower for the EUS group. Time to discharge was shortest for patients who underwent EUS $(P<$ 0.0002).

Whereas the significant differences in characteristics could have been related to the fact that fewer sites were sampled with EUS, we compared all procedures that involved sampling of two or fewer sites with each approach (none of the combined approach procedures involved sampling of fewer than two sites). Data are presented in Table 2 . There were 56 such EUS procedures and 52 such EBUS procedures, with a mean of $1.4 \pm 0.6$ sites for EUS and $1.6 \pm 0.6$ for EBUS (NS, $P=0.18$ ). All data apart from maximal oxygen flow rates were significantly different when the results from these two groups were compared $(P<0.001)$. Time to discharge remained shorter for the EUS group $(P=0.001)$.

Table 2 Data for sampling of fewer than two sites.

\begin{tabular}{|lccc|} 
& EUS $(\mathbf{n}=\mathbf{5 6})$ & EBUS $(\mathbf{n}=\mathbf{5 2})$ & $\boldsymbol{P}$ value \\
\hline Total sites & $1.4 \pm 0.6$ & $1.6 \pm 0.6$ & 0.18 \\
\hline Procedure time $(\mathrm{min})$ & $12.1 \pm 5.4$ & $21.8 \pm 8.3$ & $<0.0001$ \\
\hline Midazolam $(\mathrm{mg})$ & $3.4 \pm 1.4$ & $4.5 \pm 1.7$ & 0.0003 \\
\hline Fentanyl $(\mu \mathrm{g})$ & $72.1 \pm 27.1$ & $98.2 \pm 34.6$ & $<0.0001$ \\
\hline Maximal $\mathrm{O}_{2}$ flow $(\mathrm{L} / \mathrm{min})$ & $5.3 \pm 2.7$ & $6.4 \pm 3.4$ & 0.06 \\
\hline Time to room air $(\mathrm{min})$ & $18.6 \pm 16.9$ & $27.2 \pm 25.8$ & 0.05 \\
\hline Time to discharge $(\mathrm{min})$ & $42.4 \pm 15.8$ & $55.1 \pm 22.3$ & 0.001 \\
\hline
\end{tabular}

Abbreviations: EUS, esophageal ultrasound; EBUS, endobronchial ultrasound. 
Table 3 Nodal stations and masses sampled; listed by approach.

\begin{tabular}{|c|c|c|c|c|c|c|c|c|}
\hline \multirow[b]{2}{*}{ Site } & \multicolumn{4}{|c|}{ Single Approach } & \multicolumn{4}{|c|}{ Combined Approach } \\
\hline & EBUS & Size $(\mathrm{cm})$ & EUS & Size $(\mathrm{cm})$ & EBUS & Size $(\mathrm{cm})$ & EUS & Size $(\mathrm{cm})$ \\
\hline Station 1 & & & 1 & 1 & & & 2 & $1 \pm 0.7$ \\
\hline Station $2 \mathrm{R}$ & 4 & $2 \pm 0.5$ & 4 & $1 \pm 0.5$ & & & 1 & 0.8 \\
\hline Station $2 \mathrm{~L}$ & 1 & 1 & & & & & & \\
\hline Station 3 & 4 & $1 \pm 0.5$ & & & & & 1 & 0.8 \\
\hline Station $4 \mathrm{R}$ & 41 & $1.7 \pm 0.6$ & 3 & $3 \pm 1.5$ & 9 & $0.8 \pm 3$ & & \\
\hline Station 4L & 10 & $0.87 \pm 0.25$ & 19 & $1.35 \pm 0.9$ & & & 4 & $0.7 \pm 0.2$ \\
\hline Station 7 & 34 & $1.98 \pm 1.2$ & 45 & $2.3 \pm 1.15$ & 5 & $2.6 \pm 1.1$ & 13 & $2.3 \pm 1.9$ \\
\hline Station 8 & & & 2 & $2 \pm 1.5$ & & & & \\
\hline Station $10 R$ & 1 & 1.2 & & & 1 & 0.8 & & \\
\hline Station $10 \mathrm{~L}$ & 2 & $1.25 \pm 0.4$ & & & 1 & 0.5 & & \\
\hline Station $11 \mathrm{R}$ & 18 & $1.18 \pm 0.8$ & & & 4 & $1.25 \pm 0.5$ & & \\
\hline Station $11 \mathrm{~L}$ & 8 & $1.3 \pm 0.6$ & & & 3 & $1.4 \pm 0.8$ & & \\
\hline Station $12 \mathrm{R}$ & 3 & $0.6 \pm 0.2$ & & & 1 & 0.5 & & \\
\hline Station $13 R$ & 1 & 0.5 & & & & & & \\
\hline Mass & 19 & & 8 & & 7 & & 2 & \\
\hline Totals & 142 & & 86 & & 31 & & 23 & \\
\hline
\end{tabular}

Abbreviations: EUS, esophageal ultrasound; EBUS, endobronchial ultrasound.

Patients in which both EUS and EBUS were performed $(n=21)$ were reviewed. In twelve of these patients the sequence was EUS followed by EBUS, and in the remaining nine patients it was EBUS followed by EUS. The most common reason for a combined procedure ( $n=13,62 \%$ ) was lack of diagnosis from the first approach based upon ROSE; negative findings from one approach led to accessing other sites, or (particularly with station 7) other areas of a nodal station to be certain that a significant pathologic process had not been missed. In six of the 21 patients (28\%) the change in approach led to a positive diagnosis that was originally missed using the first approach (2/12 EUS $\rightarrow$ EBUS, 4/9 EBUS $\rightarrow$ EUS). In one patient, initial on-site EUS cytology was negative, however, a slide obtained by EUS before EBUS but processed after the change to EBUS was found to be positive for malignancy. In one patient, we switched from EBUS to EUS to obtain additional (station 7) material for flow cytometry because of patient discomfort with the endoscope in the airways.

Sites accessed are listed in Table 3. As expected, the most frequently biopsied area was station 7, which was followed by $4 \mathrm{R}$, $4 \mathrm{~L}$, and 11 R. 4 R was occasionally accessible via the esophagus. A mass/structure other than a node was accessed $13 \%$ of the time (36/282 needle aspiration biopsies). Mean nodal sizes are also listed in Table 3 although nodal size did not impact the capacity to sample it. An exception to this generalization is in $4 \mathrm{R}$ from the esophagus; it is not generally accessible from the esophagus, but in some instances it was so enlarged that esophageal access was possible. The mean number of passes was 3.42 (minimum of 3 and maximum of 10 ).

Seventy EUS procedures were performed (61 as the initial procedure, nine as crossover studies). There were two false negatives, which were documented by crossover to EBUS. In one patient, station 7 was negative from the esophagus and positive from the airway, and in a second patient, station 7 was not visualized from the esophagus, but was observed and was positive from the airway. EUS was diagnostic in the remaining $97 \%$ of patients: pathologic diagnoses among $73 \%$ and no pathology found among $23 \%$ of patients (sensitivity, 0.96; 95\%CI 0.86-0.99; specificity, $1 ; 95 \%$ CI $0.76-1)$. Negatives were shown to be negative for pathology by stability or regression over a two-year follow-up or, occasion- ally, by surgical resection. Eighty-two EBUS studies were performed. Four false negative results were documented by crossover to EUS, which yielded three station 7 nodes and one $4 \mathrm{~L}$ node. EBUS was diagnostic in the remaining 81 procedures examined, with $74 \%$ pathologic and $20 \%$ benign diagnoses (sensitivity, 0.92; 95\%CI 0.84-0.98; specificity, 1 ; $95 \%$ CI $0.74-1$ ). Once again, a benign designation was substantiated by two-year follow-up and occasionally by surgery. If the crossover studies were examined as a separate subset, diagnostic accuracy was 100\% (74\% pathologic, $26 \%$ benign, confirmed with two-year follow-up or with surgical resection).

Complications were rare. Two patients experienced severe postprocedural hypoxia (one EBUS and one EBUS+EUS). One patient had mediastinal bleeding related to the procedure (EBUS+EUS). All three of these patients were admitted for observation and discharged the following day. No interventions were required.

The primary objective of this study was to analyze differences in procedure characteristics related to differences in approach, but the data were also analyzed with respect to whether or not a specific diagnosis was achieved. Achieving a specific diagnosis did have a significant impact upon study characteristics; for all procedures combined, studies for which a diagnosis was achieved involved sampling of $1.8 \pm 0.8$ stations over $19.3 \pm 9.7 \mathrm{~min}$, whereas studies without specific diagnoses involved sampling of $2.4 \pm 0.8$ stations over $27.3 \pm 7.1 \mathrm{~min}(P=0.007$ and $P=0.017$, respectively).

\section{Discussion}

Endoscopic ultrasound has revolutionized the diagnosis of mediastinal abnormalities and the nodal staging of lung cancer. The first ultrasound endoscope to be developed was for the gastrointestinal tract [9]. EUS was initially used for the diagnostic evaluation of diseases of the gastrointestinal tract, but its application to lung cancer diagnosis and staging was rapidly conceptualized and brought to fruition [10]. The first clinical use of the smallerdiameter convex curvilinear ultrasound bronchoscope (EBUS scope) was reported in 2004 [11], and EBUS as a diagnostic tool 
has been widely adopted by the pulmonary community. It was subsequently shown both that the EBUS scope can be used for EUS and that pulmonologists can be trained to use the gastrointestinal endosonoscope $[2-5,7]$. EUS with the EBUS endoscope has not, however, been widely adopted by the pulmonary community, and prominent pulmonologists have questioned the true value of adding EUS to EBUS and stated that EBUS is the initial procedure of choice: "We agree that EBUS-TENA (their term for EUS-FNA with EBUS scope) should be used only in circumstances when lymph node stations are difficult or are not accessible by TBNA." [8] Their bottom line was that EUS using either endoscope might be better tolerated but was rarely indicated for the diagnosis of thoracic disease.

Two prospective studies have examined EBUS vs. EUS. Kang et al investigated the value of EBUS vs. EUS in the diagnosis of potentially resectable lung cancers [12]. They used the same endoscope for both procedures. Their study randomized initial approach (EBUS vs. EUS) but mandated a crossover. They found that if they did EUS first, adding EBUS contributed significantly to diagnostic yield, but if they did EBUS first there was not a significant increase, leading to the conclusion that there is no complementary role for EUS. Kang et al. were unable to comment on parameters such as sedation differences between EBUS and EUS given the mandated crossover. They concluded that EBUS is the appropriate initial approach, and the lack of complementary data from EUS would lead to the conclusion that EBUS is the only approach that should be used. They did comment in their discussion that, "EUSFNA is generally well tolerated, and procedure tolerance may affect the selection procedure."

In a second prospective study, Oki et al randomized all candidates for endosonography with lesions accessible from both airway and esophagus and performed either EBUS-TBNA alone or EUS-FNA alone while monitoring performance characteristics [13]. With both study groups sedated to the extent that there was equal patient procedure tolerance, diagnostic EUS-FNA was associated with lower doses of lidocaine $(P<0.001)$ and sedatives $(P=0.02)$, shorter procedure times $(P<0.001)$, and fewer oxygen desaturations $(P<0.001)$. Also noted was that endoscopists preferred the esophageal approach $(P<0.001)$.

Our study has the disadvantage of not being prospective/randomized and the advantage of reflecting the application of both approaches to routine clinical practice. In our laboratory, we use the same scope for both EBUS and EUS; there is no gradient of experience that leads us to choose one route over the other. It has been recurrently suggested in the literature that EUS is better tolerated than EBUS $[3,8,12,14-16]$. Our data combined with those of Oki et al provide objective confirmation for this impression. When EUS alone sufficed to achieve our clinical goals, we were able to do so with statistically significant economies of sedation, oxygen requirements, and recovery time. This was true when we looked at all diagnostic studies regardless of required sampling and remained true when we limited analysis to sampling of an equivalent number of nodes. When a crossover study was performed, total procedure time, sedation, oxygen requirements, and recovery for the combined study were significantly higher than for EUS alone but not significantly different from those for EBUS alone; there was no negative impact upon these parameters from having started with EUS.

In our study with no mandated crossover, we based the initial approach upon anatomy as defined by computed tomography scans. In so doing, we did more EBUS than EUS, reflecting the fact that more nodes were accessible via EBUS than via EUS.The numbers were not, however, dramatically different; 71 patients were diagnosed by EBUS alone and 61 by EUS alone. The major differences in sedation for the two routes occurred 1) at the time of insertion across the vocal cords and, subsequently, 2) when cough or discomfort made us wait for additional medication (systemic or endobronchial) to take effect, or 3) when patient desaturation made us pause. However, nodes are not more difficult to "see" or to reach from the airway vs. the esophagus, and identification of any specific node takes a matter of seconds. In a calm patient, one can reach $4 \mathrm{R}$ from the airway just as fast as $8 \mathrm{R}$ from the esophagus. We would thus say that nodal anatomy was not a factor. Crossover to from EUS to EBUS (or vice versa) is always an option in our lab, and in our study EBUS was just as likely as EUS to require a crossover procedure. The most important finding from the crossover data are that the two approaches were complementary; in patients not diagnosed by the initial approach, crossover led to a diagnosis $28 \%$ of the time. As noted, Kang et al. did not find EUS and EBUS to be complementary, [12] but there is a significant body of literature that, like our data, suggests that they are $[4,5,17-19]$.

This study examined needle aspiration of solid tissue structures with EUS and EBUS, and we alternated between the two approaches in some patients in both "directions" (EUS to EBUS and EBUS to EUS). The published infectious complication rate was extremely low by both routes $[20,21]$. Neither EUS nor EBUS is a "clean" procedure; the "dirtiest" structure involved in these procedures is the mouth, and both EBUS and EUS involve passing the endoscope through this bacteria-laden environment. We consider this to be the dominant etiology of risk and have felt that entry into the esophagus before the trachea does not increase that risk. Notably, aspiration of cystic structures has been associated with a higher risk [21]; we did encounter one of these situations. Based upon our understanding, we have routinely performed EBUS and EUS-FNA interchangeably, with the first procedure performed to a) maximize yield (diagnosis, staging if relevant) and b) minimize risk and discomfort. EUS-FNA is frequently the leading procedure. To date we have performed over 1000 combined procedures, and we have yet to see an infectious complication.

In summary, Villman's vision of EUS becoming a routine pulmonary procedure has not been realized; only a small fraction of the pulmonary community has incorporated EUS, some using the EBUS endoscope and an even smaller minority using the gastrointestinal endoscope. The data of Kang et al would lead to the conclusion that that EBUS alone is all that is required for evaluation of diseases involving the mediastinum [12]. The data of Oki et al coupled with our data leads to different conclusions [22]. The impression that EUS is better tolerated is now supported by data demonstrating efficiencies of sedation, time, and oxygenation with EUS vs. EBUS. This study demonstrates advantages of EUS, regardless of whether the operator is a pulmonologist or a gastroenterologist. The advantages of pulmonary alone performing both with the same endoscope include economies of time, sedation, and equipment and a seamless transition between modalities. In an institution with separate physicians performing EBUS and EUS, we would suggest careful pre-review of the radiologic data and a collaborative procedure if both routes of access are likely to yield important data. Based upon our data, we conclude that if EUS might adequately establish a diagnosis (and, if relevant, a stage), then EUS should be the first procedure. This is particularly applicable to individuals with marginal physiologic reserve. Finally, EUS and EBUS do indeed have complementary roles. Integration of the two procedures can be achieved either 
via cross-training by pulmonologists or by collaborative procedures including both pulmonology and gastroenterology.

\section{Competing interests: None}

\section{References}

1 Vilmann P, Larsen SS. Endoscopic ultrasound-guided biopsy in the chest: little to lose, much to gain. Eur Respir J 2005; 25: 400-401

2 Abouzgheib W, Nahra R, Homsi S et al. Safety and feasibility of identifying and sampling of mediastinal structures through the esophagus using EBUS. Chest 2009: DOI 10.1378/chest.136.4_MeetingsAbstracts.110S-b

3 Hwangbo B, Lee HS, Lee GK et al. Transoesophageal needle aspiration using a convex probe ultrasonic bronchoscope. Respirology 2009; 14 : $843-849$

4 Herth FJ, Krasnik M, Kahn N et al. Combined endoscopic-endobronchial ultrasound-guided fine-needle aspiration of mediastinal lymph nodes through a single bronchoscope in 150 patients with suspected lung cancer. Chest 2010; 138: 790 - 794

5 Hwangbo B, Lee GK, Lee HS et al. Transbronchial and transesophageal fine-needle aspiration using an ultrasound bronchoscope in mediastinal staging of potentially operable lung cancer. Chest 2010; 138: 795 802

6 Abouzgheib W, Shweihat Y, Bartter T. Oesophageal applications of the convex curvilinear ultrasound bronchoscope; an illustrative patient series. Respirology 2011; 16: 965 - 968

7 Annema JT, Bohoslavsky R, Burgers $S$ et al. Implementation of endoscopic ultrasound for lung cancer staging. Gastrointest Endosc 2010; 71: 64 - 70, 70 e61

8 Wang KP, Feller-Kopman D, Mehta A et al. Endobronchial ultrasound and esophageal ultrasound: just because we can, does not necessarily mean we should. Chest 2011; 140: 271 - 272; author reply 272 - 273

9 Vilmann P, Hancke S, Henriksen FW et al. Endosonographically-guided fine needle aspiration biopsy of malignant lesions in the upper gastrointestinal tract. Endoscopy 1993; 25: 523-527

10 Silvestri GA, Hoffman BJ, Bhutani MS et al. Endoscopic ultrasound with fine-needle aspiration in the diagnosis and staging of lung cancer. Ann Thorac Surg 1996; 61: 1441 - 1445; discussion 1445-1446

11 Krasnik M, Vilmann P, Larsen SS et al. Preliminary experience with a new method of endoscopic transbronchial real time ultrasound guided biopsy for diagnosis of mediastinal and hilar lesions. Thorax 2003; 58: $1083-1086$

12 Kang HJ, Hwangbo B, Lee GK et al. EBUS-centred versus EUS-centred mediastinal staging in lung cancer: a randomised controlled trial. Thorax 2013: DOI 10.1136/thoraxjnl-2013-203881

13 Oki M, Saka H, Ando $M$ et al. Transbronchial versus yransesophageal needle aspiration Uusing an ultrasound bronchoscope for the diagnosis of mediastinal lesions: a randomized study. Chest 2014: DOI $10.1378 /$ chest.14-1283

14 Medford AR, Agrawal S. Single bronchoscope combined endoscopic-endobronchial ultrasound-guided fine-needle aspiration for tuberculous mediastinal nodes. Chest 2010; 138: 1274

15 Araya T, Demura Y, Kasahara $K$ et al. Usefulness of transesophagea bronchoscopic ultrasound-guided fine-needle aspiration in the pathologic and molecular diagnosis of lung cancer lesions adjacent to the esophagus. J Bronchology Interv Pulmonol 2013; 20: 121 - 126

16 Annema JT, Rabe KF. Endosonography for lung cancer staging: one scope fits all? Chest 2010; 138: 765 - 767

17 Vilmann P, Krasnik M, Larsen SS et al. Transesophageal endoscopic ultrasound-guided fine-needle aspiration (EUS-FNA) and endobronchial ultrasound-guided transbronchial needle aspiration (EBUS-TBNA) biopsy: a combined approach in the evaluation of mediastinal lesions. Endoscopy 2005; 37: 833-839

18 Rintoul RC, Skwarski KM, Murchison JT et al. Endobronchial and endoscopic ultrasound-guided real-time fine-needle aspiration for mediastinal staging. Eur Respir J 2005; 25: 416-421

19 Wallace MB, Pascual JM, Raimondo $M$ et al. Minimally invasive endoscopic staging of suspected lung cancer. JAMA 2008; 299: 540-546

20 Polkowski M, Larghi A, Weynand B et al. Learning, techniques, and complications of endoscopic ultrasound (EUS)-guided sampling in gastroenterology: European Society of Gastrointestinal Endoscopy (ESGE) Technical Guideline. Endoscopy 2012; 44: 190 - 206

21 von Bartheld MB, van Breda A, Annema JT. Complication rate of endosonography (endobronchial and endoscopic ultrasound): a systematic review. Respiration 2014; 87: 343-351

22 Oki M, Saka H, Kitagawa C et al. Prospective study of endobronchial ultrasound-guided transbronchial needle aspiration of lymph nodes versus transbronchial lung biopsy of lung tissue for diagnosis of sarcoidosis. J Thorac Cardiovasc Surg 2012; 143: 1324-1329 